\title{
Anti-diabetic and anti-obesity effects of the allosteric PTP1B inhibitor KY-226 via insulin and leptin signal enhancement
}

\author{
Yuma Ito ${ }^{1,2}$, Masaki Fukui ${ }^{1}$, Tatsuya Kitao ${ }^{1}$, Mamoru Kanda ${ }^{1}$, Eiichi Hinoi ${ }^{2}$, Hiroaki Shirahase ${ }^{1}$ \\ ${ }^{1} R \& D$ Division, Kyoto Pharmaceutical Industries, Ltd., Japan, ${ }^{2}$ Laboratory of Molecular Pharmacology, Division of \\ Pharmaceutical Sciences, Kanazawa University Graduate School, Japan
}

Background: PPAR $\gamma$ agonists have been used as insulin sensitizers for the treatment of type 2 diabetes; however, they also exert a number of adverse effects. Therefore, the development of PPAR $\gamma$-independent insulin sensitizers is desired. Since protein tyrosine phosphatase 1B (PTP1B) is a negative regulator of insulin and leptin signaling, PTP1B inhibitors have potential as anti-diabetic and anti-obesity drugs. We previously reported the novel non-competitive allosteric PTP1B inhibitor 4-(biphenyl-4-ylmethylsulfanylmethyl)-N-(hexane-1-sulfonyl) benzoylamide (KY-226). In the present study, we pharmacologically characterized KY-226 and investigated its effects on experimental diabetes and obesity.

Methods: The PTP1B inhibitory activity, PPAR $\gamma$ agonist activity, and effects of KY-226 on adipocyte differentiation in 3T3-L1 cells and insulin-induced phosphorylation in HepG2 cells were examined. The effects of KY-226 on diabetes via insulin signal enhancement in $d b / d b$ mice on obesity and via leptin signal enhancement in diet-induced obesity (DIO) mice were also investigated.

Results: KY-226 inhibited PTP1B activity $(\mathrm{IC} 50=0.28 \mu \mathrm{M})$ and potentiated the insulin-induced phosphorylation of insulin receptor (IR) in HepG2 cells. KY-226 did not exhibit PPAR $\gamma$ agonist activity and had no significant effects on adipocyte differentiation, whereas the PPAR $\gamma$ agonist pioglitazone significantly enhanced it. In $d b / d b$ mice, KY-226 reduced plasma glucose levels at $30 \mathrm{mg} / \mathrm{kg} /$ day (p.o., 4 weeks) and TG and HbA1c levels at 10 and $30 \mathrm{mg} / \mathrm{kg} /$ day without increasing body weight, while pioglitazone exerted similar diabetic effects with elevations in body weight. In the oral glucose tolerance test, KY-226 significantly decreased elevated plasma glucose levels. KY-226 enhanced the insulininduced phosphorylation of IR and Akt in the liver and femoral muscle. In DIO mice, KY-226 decreased total food consumption at $60 \mathrm{mg} / \mathrm{kg} /$ day, and body weight gain and fat volume gain at 30 and $60 \mathrm{mg} / \mathrm{kg} / \mathrm{day}$. KY-226 also potentiated the leptin-induced phosphorylation of STAT3 in the hypothalamus.

Conclusions: These results demonstrate that KY-226 exerts anti-diabetic and anti-obesity effects by enhancing insulin and leptin signaling, respectively, without increasing body weight. Thus, KY-226 is a promising candidate for an efficacious and safe anti-diabetic drug with anti-obesity effects. 